\title{
Diagnosis, Staging, and Patient Selection for Locoregional Therapy to Treat Hepatocellular Carcinoma
}

\author{
Zachary T. Berman, MD ${ }^{1}$ Isabel Newton, MD, $\mathrm{PhD}^{1,2}$ \\ ${ }^{1}$ Department of Radiology, University of California San Diego, San \\ Diego, California \\ 2 Department of Radiology, Veterans Affairs San Diego Healthcare \\ System, San Diego, California \\ Address for correspondence Zachary T. Berman, MD, Department of \\ Radiology, University of California San Diego, 200 W. Arbor Dr., San \\ Diego, CA 92103 (e-mail: ZBerman@health.ucsd.edu).
}

Semin Intervent Radiol 2020;37:441-447

\begin{abstract}
Keywords

- hepatocellular carcinoma

- staging

- locoregional therapy

Hepatocellular carcinoma (HCC) is the second leading cause of cancer-related mortality and the only cancer for which the incidence and mortality are on the rise. Sensitive and specific screening and diagnostic approaches, robust staging regimens, multidisciplinary tumor boards, and patient/family education and engagement in the shared decision-making process help to identify a patient's optimal treatment options. Locoregional therapies have been the mainstay for treating intermediate-stage disease, but they are finding special applications for early and advanced disease. This review discusses the diagnosis of HCC, current accepted staging models, and treatment of HCC, with a focus on locoregional therapies.
\end{abstract}

Hepatocellular carcinoma (HCC) is the fifth leading cause of cancer and the second leading cause of cancer mortality worldwide. ${ }^{1,2}$ According to the Centers for Disease Control and Prevention, liver cancer is the only cancer for which the incidence and mortality are rising: between 2003 and 2012, the incidence of liver cancer increased by $38 \%$ and the mortality increased by $56 \%{ }^{3}$ The incidence has almost tripled in the last three decades in the United States and is expected to continue to rise through 2030 with the rise in obesity and nonalcoholic steatohepatitis (NASH). ${ }^{4}$ The African American and Hispanic communities are expected to experience the highest growth in the United States. ${ }^{5}$ In Asia and Africa, the leading cause of HCC is chronic hepatitis B virus (HBV) infection and aflatoxin exposure, while in the United States hepatitis $\mathrm{C}$ virus (HCV) infection is the leading cause, with alcoholic cirrhosis and NASH/obesity as growing factors. ${ }^{6}$ Without treatment, the median survival of HCC is 6 to 20 months, and the 5 -year survival is approximately 10 to $14 \%$. Treatment options vary significantly by disease stage, and outcomes are better with early diagnosis and treatments that can cure or curb the disease. Thus, accurate diagnosis and staging remain critical for providing the appropriate treatment for patients. ${ }^{6}$
This review will describe current accepted diagnosis paradigms, discuss treatment options with a focus on locoregional therapies, and describe the ideal candidates for locoregional therapies vis-a-vis the currently accepted and proposed staging models, which will be critically evaluated. Lastly, we will review methods for assessing response after locoregional therapy to determine when repeat treatment is indicated.

\section{Surveillance and Diagnosis}

HCC develops in a setting of chronic inflammation due to hepatitis B or C viral infection, ethanol damage, $\mathrm{NASH}$, and other etiologies. ${ }^{7}$ Given the long latency period for the development of HCC, various screening protocols have been established to allow for early detection, when patients may still be eligible for curative treatments. The American Association for the Study of Liver Diseases (AASLD) and the European Association for the Study of the Liver (EASL) recommend surveillance for adult patients at risk for developing HCC (Level 1A evidence). ${ }^{8,9}$ The risk of developing HCC is dependent on the underlying etiology of liver inflammation and the presence of cirrhosis. For instance, the incidence of HCC in Asian men with
Issue Theme Seminars in IR Liver Oncology; Guest Editor, Siddharth A. Padia, MD
Copyright (C) 2020 by Thieme Medical Publishers, Inc., 333 Seventh Avenue, New York, NY 10001, USA. Tel: +1(212) 760-0888.
DOI https://doi.org/ 10.1055/s-0040-1719185. ISSN 0739-9529. 
$\mathrm{HBV}$ is 0.3 to $0.6 \%$ per year if there is no cirrhosis but 3 to $5 \%$ per year if cirrhosis is present. ${ }^{8}$ Therefore, the recommendation is to screen patients with cirrhosis of any cause or HBV without cirrhosis in Asian and African American men older than 40 years and Asian women older than 50 years. ${ }^{10}$

In most countries, surveillance is performed with ultrasound, with or without the addition of serum $\alpha$-fetoprotein (AFP) levels (which is not always elevated in HCC). ${ }^{6}$ One Chinese randomized controlled trial (RCT) demonstrated that screening with ultrasound and AFP can lead to a $37 \%$ reduction in mortality. ${ }^{11}$ In the United States, initial surveillance is also often performed with multiphase computed tomography (CT) or magnetic resonance imaging (MRI), with the advantage of being less operator dependent but for much greater expense. Cost effectiveness studies have been done which confirm a benefit of HCC screening and surveillance with ultrasound. ${ }^{12}$ No RCT has been done to evaluate the sensitivity or specificity of surveillance in patients with HCV or other causes of cirrhosis, although given the current clinical standard of surveillance in this population, the feasibility of being able to conduct an RCT in this cohort is logistically and possibly ethically questionable. ${ }^{13}$ There has been growing acceptance of contrast-enhanced ultrasound (CEUS) to diagnose HCC. A recent meta-analysis concluded that CEUS has a sensitivity of $85 \%$ and a specificity of $91 \%$ for detecting HCC. ${ }^{14}$ However, the AASLD recommends larger scale studies to confirm these findings before widely recommending CEUS for HCC detection. ${ }^{8}$

Once surveillance ultrasound identifies a lesion suspicious for liver cancer, both AASLD and EASL recommend further imaging with multiphasic CT or MRI. ${ }^{8,9}$ The AASLD recommends diagnosis based on the American College of Radiology's (ACR's) Liver Imaging Reporting and Data System (LI-RADS; -Fig. 1), whereas EASL recommends basing diagnosis on their own criteria. Arterial phase hyperenhancement and portal venous/delayed phase washout remain the hallmarks of HCC for both LI-RADS and EASL (- Fig. 2). If a lesion does not demonstrate classic features of HCC, then biopsy is recommended to guide treatment. Likewise, if a lesion is suspicious for HCC on imaging in a patient without cirrhosis or underlying liver disease (e.g., HCV, HBV, or NAFLD) then biopsy is also indicated to establish the diagnosis.

\section{Staging}

Treatments for HCC vary by stage and degree of hepatic decompensation from cirrhosis, and treatment in the early stage can be potentially curative. In the intermediate and advanced stages, locoregional and systemic therapies can be offered with the goal of downstaging to transplantation, bridging to transplantation, controlling disease, or palliation. Categorizing HCC patients into stages helps guide the treatment choice (-Fig. 3 ) to maximize tumor response while minimizing adverse outcomes and potentially futile or morbid interventions.

The TNM staging system is not regularly used to guide liver cancer treatment decisions because it does not take into account factors which impact prognosis and treatment response, namely, liver function and patient's performance status (PS; Eastern Cooperative Oncology Group 0-5). HCCspecific staging systems that incorporate these factors include the Barcelona Clinic Liver Cancer (BCLC) staging system and Hong Kong Liver Cancer (HKLC) staging system; less commonly used are the Okuda staging system, Japanese integrated score, and Italian Liver Cancer staging system. ${ }^{15}$ The BCLC and HKLC systems both take into account the tumor burden, PS, and liver function as measured by the Child-Pugh (CP) score A-C.

The AASLD and EASL recommend staging HCC patients using the BCLC system, which is composed of stages $0-D^{8,9}$ Very early and early stages $0-A$ include patients with low tumor burden ( $\leq 3$ tumors, $<3 \mathrm{~cm}$ ) and preserved liver function (CPA) and PS (0), with a corresponding life expectancy of $>5$ years. The intermediate stage includes multifocal disease, maintained liver function (CP A-B7), and preserved PS (0), with a life expectancy of approximately 2.5 years. Advanced disease includes symptomatic, invasive, or metastatic disease with preserved liver function but worsening PS (1-2); life expectancy is greater than 10 months. End-stage disease includes any tumor volume with poor liver function (CP C) or PS $>2$; life expectancy is only 3 months. ${ }^{9}$ The widespread adoption of the BCLC system is due to its simplicity and reproducibility with respect to prognosis for each stage. However, it has been critiqued as being overly conservative particularly in the intermediate and advanced stage settings. To better predict prognosis and capture patients who would

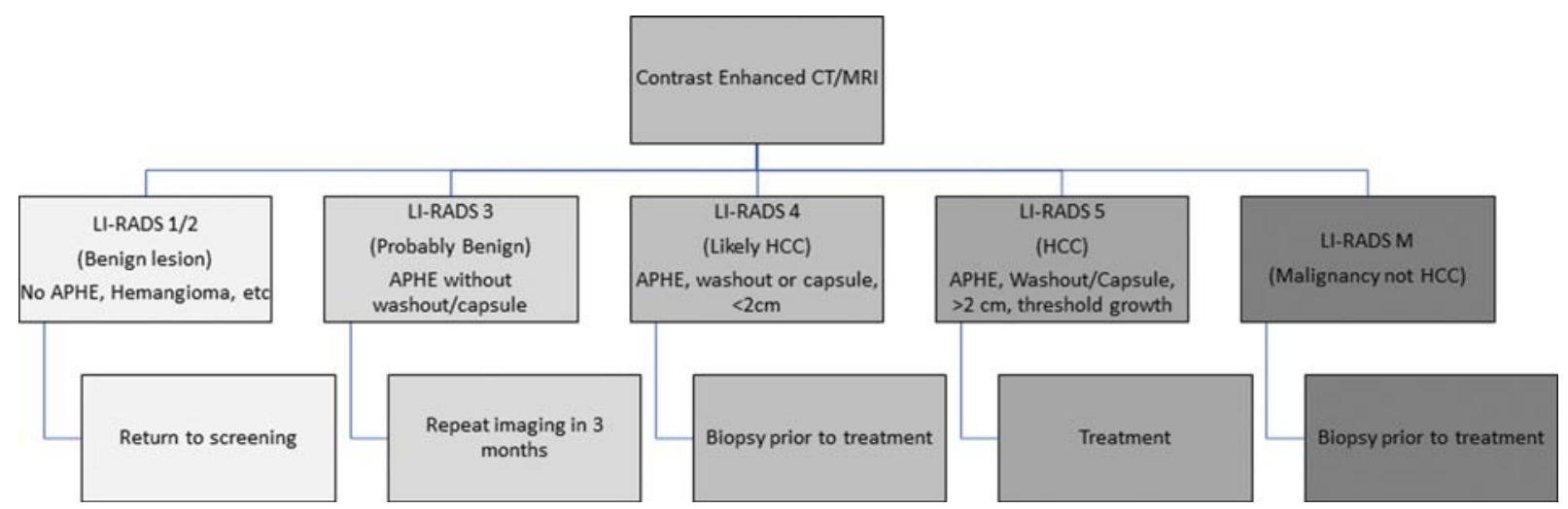

Fig. 1 Flow chart demonstrating the different categories of findings on multiphasic liver computed tomography or magnetic resonance imaging with next management steps. 


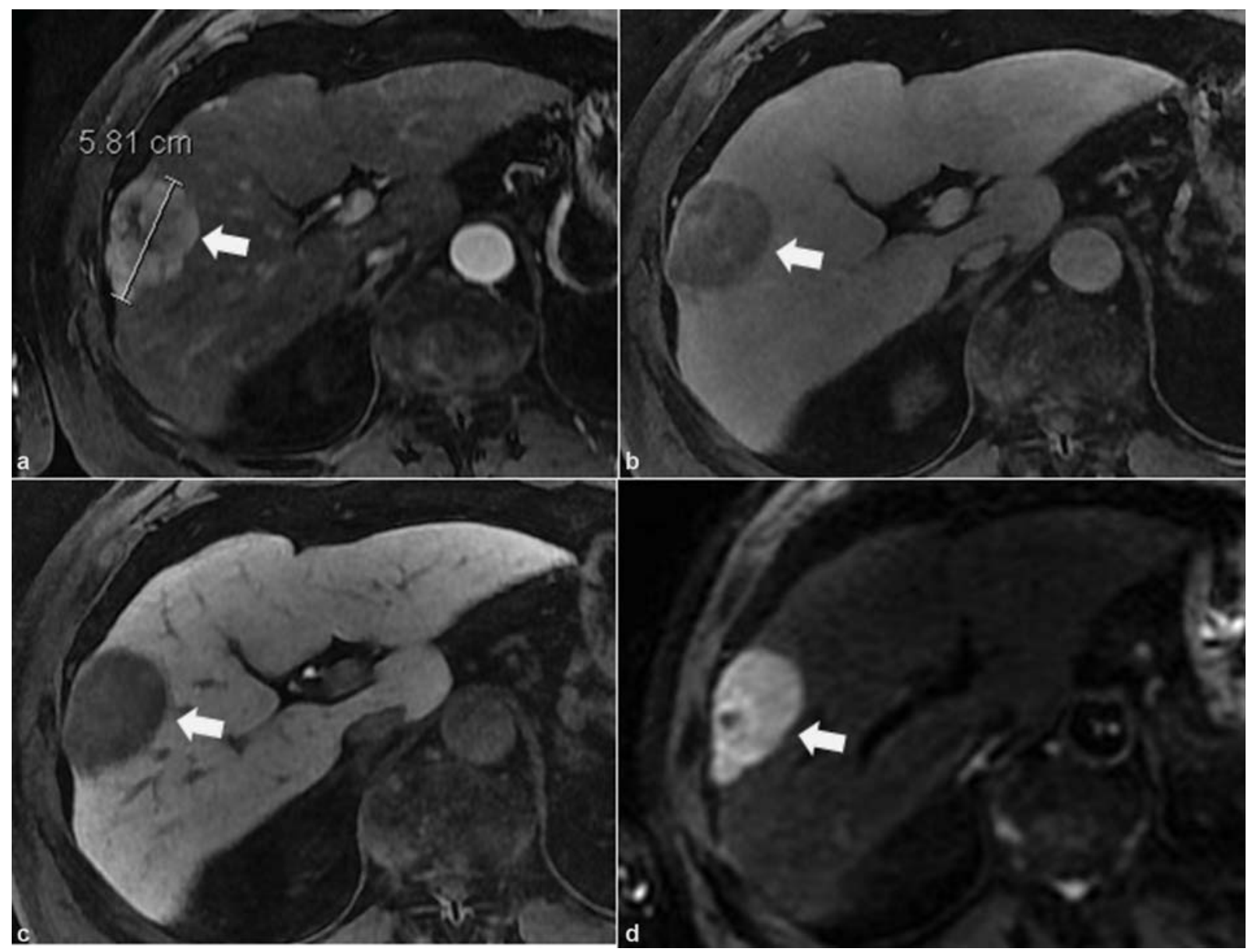

Fig. 2 Common findings of HCC on multiphasic MRI after administration of a hepatobiliary contrast agent (gadoxetic acid). (a) Arterial phase axial T1-weighted fat-saturated image demonstrating an arterially hyperenhancing lesion in segment 8 which washes out on delayed and hepatobiliary sequences (b, c) (white arrows). (d) The lesion (white arrow) demonstrates restricted diffusion on a DWI sequence. DWI, diffusionweighted imaging; HCC, hepatocellular carcinoma; MRI, magnetic resonance imaging.

benefit from locoregional treatments and other more aggressive therapies, the HKLC system was developed. The HKLC system consists of nine stages and offers more patient outcome stratification at the cost of increased complexity. ${ }^{9}$ The BCLC and HKLC methods can be integrated into conceptual early, intermediate/advanced, and end-stage disease (-Fig. 3; patient examples in -Fig. 4).

A common weakness of both the BCLC and HKLC systems is their dependence on the $\mathrm{CP}$ system for cirrhosis, which relies on the subjective assessments of encephalopathy and ascites. To develop a more objective measure of liver function, the ALBI score was developed based on serum albumin and bilirubin levels. The ALBI score has been validated in patients after liver resection, TARE, and treatment with sorafenib. ${ }^{16-18}$ Currently, the AASLD/EASL makes no recommendation for the usage of the ALBI score, but ongoing studies are aimed at improving staging with the use of objective criteria.

\section{Hepatocellular Carcinoma Treatment by Stage}

If a patient has early-stage HCC (BCLC 0/A, HKLC I/II), a curative approach may be pursued through transplantation, resection, or ablation. Liver transplantation remains the gold standard curative approach, but the scarcity of transplantable organs and stringent pretransplant health and social requirements make it an untenable option for most patients. In the nontransplant setting, both the AASLD and EASL recommend resection and thermal ablation (RFA, MWA, cryoablation) as first-line therapies for the treatment of early-stage disease, with the goal of a $75 \% 5$-year survival. ${ }^{8,9}$ RCTs have demonstrated an advantage of thermal ablation over percutaneous ethanol ablation, particularly for lesions greater than $2 \mathrm{~cm} .{ }^{19,20}$ A recent meta-analysis showed similar outcomes between resection and thermal ablation, with the latter being less expensive and less morbidity. ${ }^{21,22}$ When thermal ablation is not feasible due to potential thermal damage to surrounding critical structures (biliary ducts, gallbladder, bowel, etc.), irreversible electroporation, a nonthermal ablative technique, has been shown to be effective and safe. ${ }^{23}$ TARE has recently been proposed as a potentially curative option due to retrospective studies showing median overall survival of approximately 6.7 years and a 5-year survival of $75 \% .{ }^{24}$ Additional prospective trials are needed to validate these findings. Patients with earlystage disease usually spend longer than 6 months on the transplant waiting list; therefore, it is recommended to perform locoregional therapies for listed patients as a bridge to 


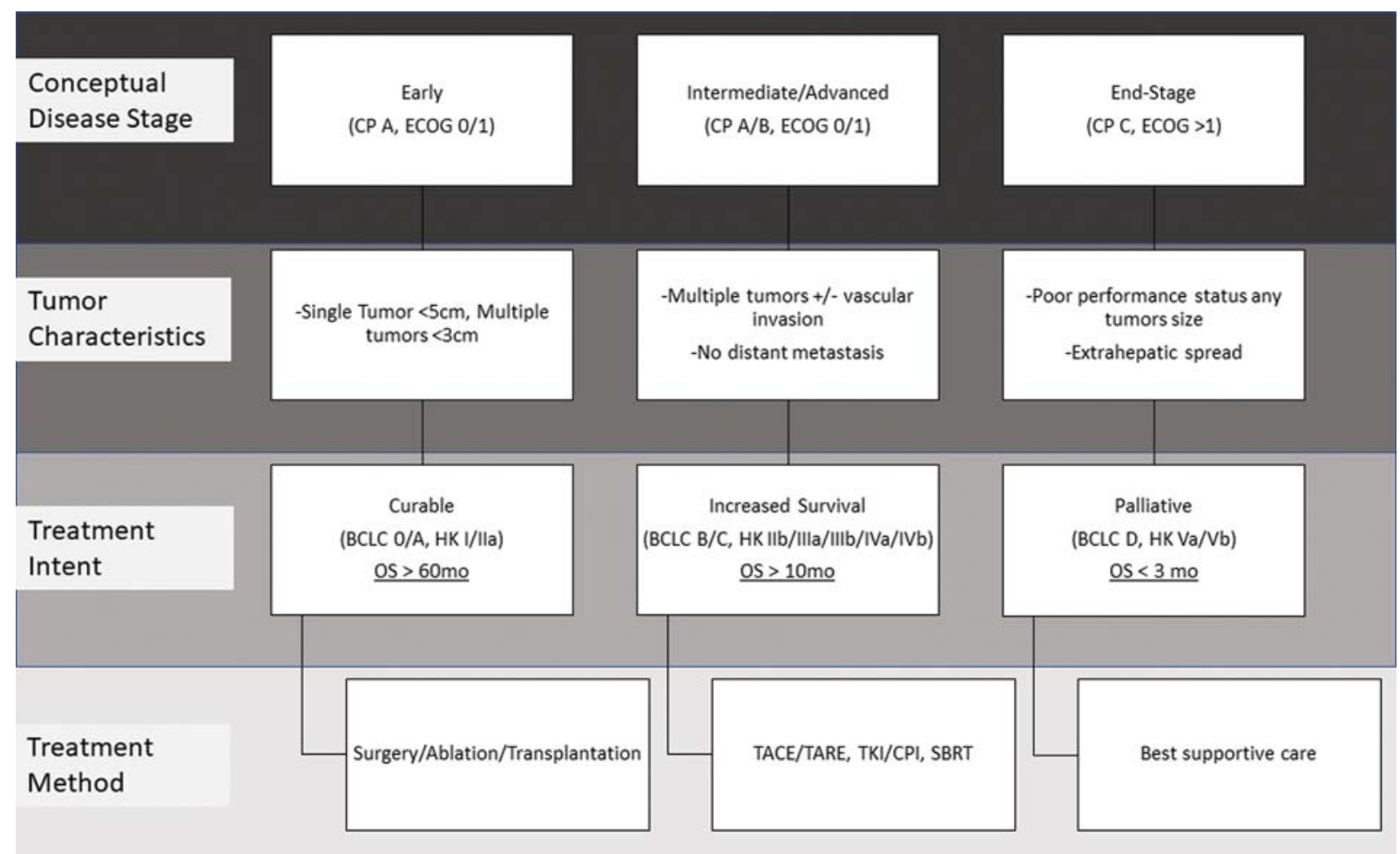

Fig. 3 Flow chart describing a staging approach to evaluate patients for locoregional therapy with corresponding staging according to the Barcelona Liver Clinic system (BCLC) and Hong Kong Liver Clinic system (HKLC). CP, Child-Pugh; PS, performance status; OS, overall survival; TACE, transarterial chemoembolization; TARE, transarterial radioembolization, TKI/CPI, tyrosine kinase inhibitor/check point inhibitor; SBRT, stereotactic body radiotherapy.
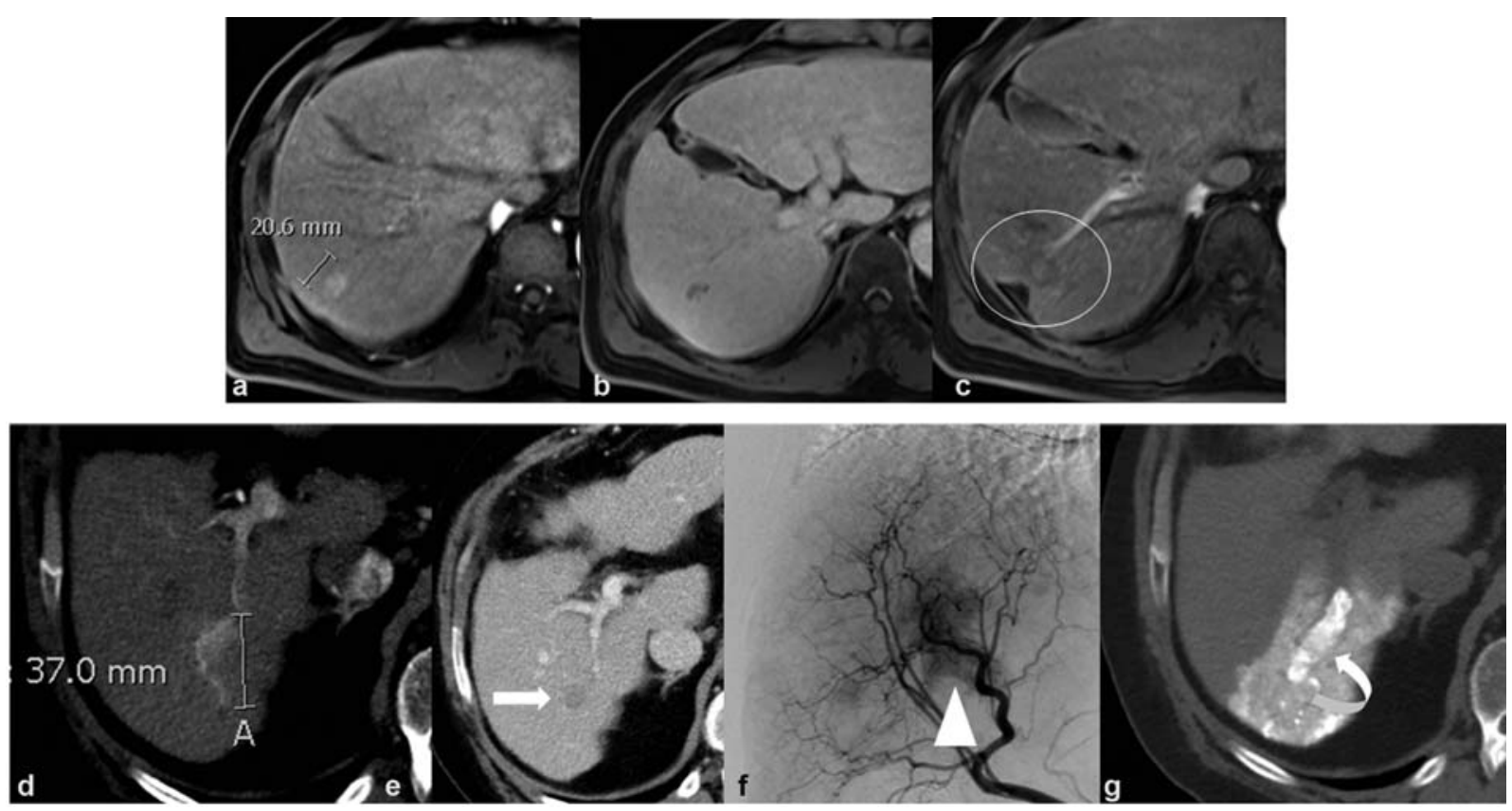

Fig. 4 Two representative patients presenting with HCC. Patient 1 has a 20.6-mm arterial phase hyperenhancing (a) lesion with washout (b) on MRI. Given their CP A and PS 0 status, they were treated with percutaneous thermal ablation. MRI 2 years later (c) demonstrates no residual arterial phase hyperenhancement with intrinsic T1-weighted hyperintense consistent with blood products in a nonviable ablation cavity (circle). Patient 2 has a 37-mm arterial hyperenhancing lesion with washout (white arrow) on delayed phases on contrast-enhanced CT (d, e). Given the CP B score, they were treated with TACE. (f) Representative digitally subtracted angiography of the right hepatic artery during the TACE procedure demonstrating tumor blush with a component that is tubular (arrowhead). (g) Non-contrast-enhanced CT after TACE demonstrating dense lipiodol uptake in the right posterior portal vein consistent with tumor invasion, which was not noted prior to the procedure. Given the progression to advanced disease, the patient was transitioned to systemic therapy. CP, Child-Pugh; CT, computed tomography; HCC, hepatocellular carcinoma; MRI, magnetic resonance imaging; PS, performance status; TACE, transarterial chemoembolization. 
transplantation. ${ }^{8}$ Although surgery and ablation represent potentially curative treatments for early-stage disease, most patients present with intermediate-stage disease, for which locoregional therapies represent the best options for disease control.

Locoregional interventions for intermediate-stage disease (BCLC B/C, HKLC IIb/IIIa/IIIb/IVa/IVb) are aimed at prolonging survival. Currently, treated intermediate and advanced stage patients have an estimated survival of up to 26 and 10 months, respectively. ${ }^{9,25}$ For patients with BCLC stage B disease, RCTs and meta-analyses have demonstrated a survival benefit of locoregional therapies, making them the current recommendation of AASLD/EASL. ${ }^{8,9,25,26}$ Catheterbased locoregional therapies include TAE with bland embolic particles; conventional TACE (CTACE) with lipiodol mixed with chemotherapeutic agents, plus or minus an embolic material; drug-eluting bead TACE (DEB-TACE), where particles are conjugated to a chemotherapeutic agent; or TARE with Yttrium-90 eluting microparticles. RCTs and metaanalyses debate the efficacy of CTACE versus TAE versus DEB-TACE. ${ }^{27,28}$ The largest RCT comparing CTACE to DEBTACE, the PRECISION V trial, did not demonstrate a difference between the two treatments, although subgroup analysis showed potentially increased response rates for DEB-TACE with decreased liver toxicity in CP B, ECOG 1 , and patients with bilobar disease or recurrent disease. ${ }^{29} \mathrm{~A}$ RCT comparing DEB-TACE to TAE showed similar outcomes, suggesting that the main mechanism for cellular death is ischemia rather than by the chemotherapeutic agent. ${ }^{27}$ This finding is further supported by noting the outcomes from cTACE are independent of the type or number of chemotherapeutic agents used, which suggests that these agents confer little to no additional tumoricidal benefit. ${ }^{30}$ Given the conflicting evidence surrounding which version of TACE is superior, the choice usually depends on operator and institutional preference. However, if future percutaneous ablation or SBRT is planned, CTACE or DEB-TACE plus lipiodol can be done to render the tumor radiopaque for use as a target or fiducial. The addition of lipiodol can be particularly useful for tumors between 3 and $7 \mathrm{~cm}$, where the combination of TACE and MWA has been shown to confer additional disease control rates and overall survival. ${ }^{31-33}$

While not currently recommended by the societies for BCLC B patients, a meta-analysis of studies comparing TACE to TARE demonstrated similar 1-year overall survival (OS) but improved OS at 2 and 3 years with TARE. ${ }^{34}$ Additionally, a phase II RCT study evaluating CTACE versus TARE in early to intermediate stage patients showed a significantly increased time to progression, with a difference of at least 22 months, suggesting a potential benefit for TARE in this patient population, although overall survival was similar between the groups. ${ }^{35}$ More data are needed to validate these findings before TARE can be recommended over TACE for this population. Another limitation for TARE is the need for a total bilirubin less than $2 \mathrm{mg} / \mathrm{dL}$ when performing lobar treatment where TACE can usually be done safely up to less than $3 \mathrm{mg} / \mathrm{dL}$. In select intermediate-stage patients, locoregional therapies can also be considered to downstage patients back into transplantation criteria. In a prospective case series involving 118 patients, 77 were able to be downstaged to transplantation criteria and $54 \%$ were eventually transplanted. $^{36}$

For advanced (BCLC C) disease, recent trials have demonstrated modest increased survival with the use of systemic agents, including tyrosine kinase inhibitors (sorafenib, lenvatinib, regorafenib). ${ }^{37}$ New research shows modest responses in HCC patients with check point inhibitors targeting PD-1/PD-L1 receptors, such as pembrolizumab, nivolumab, cemiplimab, and atezolizumab. The recent RCT of atezolizumab and bevacizumab versus sorafenib showed improved 1-year survival and overall response rate $(67.2$ vs. $54.6 \%$ and 27 vs. $12 \%$, respectively). ${ }^{38}$ Two RCTs evaluated TACE with the addition of systemic agents (brivanib or sorafenib) for intermediate-stage disease and found no additional OS benefit with combination therapy. ${ }^{39,40}$

Locoregional therapy is not routinely recommended for patients with advanced-stage disease. These patients may have portal vein thrombosis or other tumoral vascular invasion which may render embolizing procedures like TACE, TAE, and DEB-TACE more dangerous, given the greater risk of infarction. Nonetheless, retrospective studies combing TACE with systemic therapies in advanced-stage disease show promising results in select patients, with longer time to progression and overall survival. ${ }^{41-45}$ The SARAH and SIRveNIB trials studied the role of TARE and did not demonstrate an overall survival difference between TARE and sorafenib for locally advanced disease, although quality of life and tumor response rates were improved with TARE. ${ }^{46,47}$ Ongoing trials are evaluating the combination of TARE and sorafenib for HCC (e.g., STOP-HCC). A recent RCT assessing boosted doses of TARE with ablative intent $(>205 \mathrm{~Gy}$ ) in intermediate- and advanced-staged patients with tumors greater than $10 \mathrm{~cm}$, many with portal vein thrombosis, has also shown promising overall survival improvements of approximately 20 months. ${ }^{48}$

Currently, there is no role for surgery (other than transplantation) or minimally invasive, image-guided procedures for the treatment of end-stage HCC (BCLC D, HKLC V). Management is mostly directed at symptom control rather than prolonging life. Interventional radiologists offer supportive procedures such as paracentesis, peritoneal tunneled drainage catheter placement, and port placement to assist with easier venous access for medication administration.

Given the different staging systems and variety of treatment options alone and in combination, HCC treatment recommendations are best made by a multidisciplinary tumor board. This team typically includes members from interventional radiology, hepatology, transplant surgery, medical oncology, radiation oncology, and diagnostic radiology. This team approach facilitates consideration of the full range of options and limitations within the context of a patient's overall treatment goals. Before and after the tumor board makes a recommendation, the patient should be seen in an outpatient consultation. This interaction offers an opportunity to educate the patient regarding their options and to exercise their autonomy in eliciting their preferences. 
This visit may exclude some treatment options due to patient preference, lack of availability close to the patient, or cost/lack of insurance coverage. Some systemic agents, SBRT, and TARE can be cost prohibitive for some patients. In these cases, a different treatment modality (e.g., TACE) may be favored. The clinic visit also serves to open a line of dialogue with the patients and their family about realistic expectations of potential outcomes. Moreover, it paves the way for future end-of-life discussions if the patient becomes ineligible for additional treatment.

\section{Treatment Evaluation}

Follow-up with imaging is critical to assess the treatment efficacy and determine when further treatment is indicated. Follow-up is usually obtained 1 to 3 months after the first treatment and then every 3 months after locoregional therapies or up to every 6 months after successful ablation or resection. LI-RADS defines criteria for the assessment of residual/recurrent disease. Viable disease is described by nodular, mass-like, or thick irregular tissue in or along the treated lesion with arterial phase hyperenhancement, washout, or a similar enhancement pattern prior to treatment. ${ }^{49}$ The degree of tumor response is measured with the AASLDrecommended modified Response Evaluation Criteria in Solid Tumors (mRECIST) or the EASL classification. ${ }^{8,9}$ For recurrent or residual disease, the patient should be presented again at the multidisciplinary conference for determination for the next best treatment option (e.g., repeat same intervention after TACE, switch modalities to TARE or SBRT, or continue surveillance). Practice patterns vary widely with regard to the number of times a modality will be attempted before it is deemed to be ineffectual or insufficient for a certain patient, and the data do not support one approach over another.

\section{Conclusions}

The successful management of patients with HCC depends on accurate and early diagnosis, the use of staging systems that predict both prognosis and the appropriate treatment, and multidisciplinary discussions that incorporate patient preference. Locoregional therapies remain the mainstay for intermediate-stage disease, both at diagnosis and for recurrent disease.

Conflicts of Interest

The authors have no conflicts of interest to disclose.

\section{References}

1 Jemal A, Bray F, Center MM, Ferlay J, Ward E, Forman D. Global cancer statistics. CA Cancer J Clin 2011;61(02):69-90

2 Ferlay J, Soerjomataram I, Dikshit R, et al. Cancer incidence and mortality worldwide: sources, methods and major patterns in GLOBOCAN 2012. Int J Cancer 2015;136(05):E359-E386

3 United States cancer statistics: 1999-2013 Incidence and mortality web-based report US Cancer Statistics Working Group US Department of Health and Human Services, CDC, National Cancer Institute Published2016
4 El-Serag HB, Kanwal F. Epidemiology of hepatocellular carcinoma in the United States: where are we? Where do we go?. Hepatology 2014;60(05):1767-1775

5 Petrick JL, Kelly SP, Altekruse SF, McGlynn KA, Rosenberg PS. Future of hepatocellular carcinoma incidence in the United States forecast through 2030. J Clin Oncol 2016;34(15):1787-1794

6 McGlynn KA, London WT. The global epidemiology of hepatocellular carcinoma: present and future. Clin Liver Dis 2011;15(02): 223-243, vii-x

7 Sia D, Villanueva A, Friedman SL, Llovet JM. Liver cancer cell of origin, molecular class, and effects on patient prognosis. Gastroenterology 2017;152(04):745-761

8 Marrero JA, Kulik LM, Sirlin CB, et al. Diagnosis, staging, and management of hepatocellular carcinoma: 2018 practice guidance by the American Association for the Study of Liver Diseases. Hepatology 2018;68(02):723-750

9 European Association for the Study of the Liver Electronic address: easloffice@easloffice.eu European Association for the Study of the Liver. EASL Clinical Practice Guidelines: management of hepatocellular carcinoma. J Hepatol 2018;69(01):182-236

10 Fetzer DT, Rodgers SK, Harris AC, et al. Screening and surveillance of hepatocellular carcinoma: an introduction to ultrasound liver imaging reporting and data system. Radiol Clin North Am 2017;55 (06):1197-1209

11 Zhang BH, Yang BH, Tang ZY. Randomized controlled trial of screening for hepatocellular carcinoma. J Cancer Res Clin Oncol 2004;130(07):417-422

12 Ruggeri M. Hepatocellular carcinoma: cost-effectiveness of screening. A systematic review. Risk Manag Healthc Policy 2012;5:49-54

13 Poustchi H, Farrell GC, Strasser SI, Lee AU, McCaughan GW, George J. Feasibility of conducting a randomized control trial for liver cancer screening: is a randomized controlled trial for liver cancer screening feasible or still needed? Hepatology 2011;54(06): 1998-2004

14 Zhang J, Yu Y, Li Y, Wei L. Diagnostic value of contrast-enhanced ultrasound in hepatocellular carcinoma: a meta-analysis with evidence from 1998 to 2016. Oncotarget 2017;8(43):75418-75426

15 Marrero JA, Kudo M, Bronowicki JP. The challenge of prognosis and staging for hepatocellular carcinoma. Oncologist 2010;15 (Suppl 4):23-33

16 Wang YY, Zhong JH, Su ZY, et al. Albumin-bilirubin versus ChildPugh score as a predictor of outcome after liver resection for hepatocellular carcinoma. Br J Surg 2016;103(06):725-734

17 Ogasawara S, Chiba T, Ooka Y, et al. Liver function assessment according to the Albumin-Bilirubin (ALBI) grade in sorafenibtreated patients with advanced hepatocellular carcinoma. Invest New Drugs 2015;33(06):1257-1262

18 Gui B, Weiner AA, Nosher J, et al. Assessment of the albuminbilirubin (ALBI) grade as a prognostic indicator for hepatocellular carcinoma patients treated with radioembolization. Am J Clin Oncol 2018;41(09):861-866

19 Germani G, Pleguezuelo M, Gurusamy K, Meyer T, Isgrò G, Burroughs AK. Clinical outcomes of radiofrequency ablation, percutaneous alcohol and acetic acid injection for hepatocelullar carcinoma: a meta-analysis. J Hepatol 2010;52(03):380-388

20 Cho YK, Kim JK, Kim MY, Rhim H, Han JK. Systematic review of randomized trials for hepatocellular carcinoma treated with percutaneous ablation therapies. Hepatology 2009;49(02): 453-459

21 Cucchetti A, Piscaglia F, Cescon M, et al. Cost-effectiveness of hepatic resection versus percutaneous radiofrequency ablation for early hepatocellular carcinoma. J Hepatol 2013;59(02):300-307

22 Cho YK, Kim JK, Kim WT, Chung JW. Hepatic resection versus radiofrequency ablation for very early stage hepatocellular carcinoma: a Markov model analysis. Hepatology 2010;51(04): 1284-1290 
23 Sutter O, Calvo J, N'Kontchou G, et al. Safety and efficacy of irreversible electroporation for the treatment of hepatocellular carcinoma not amenable to thermal ablation techniques: a retrospective single-center case series. Radiology 2017;284(03): 877-886

24 Lewandowski RJ, Gabr A, Abouchaleh N, et al. Radiation segmentectomy: potential curative therapy for early hepatocellular carcinoma. Radiology 2018;287(03):1050-1058

25 Llovet JM, Bruix J. Systematic review of randomized trials for unresectable hepatocellular carcinoma: chemoembolization improves survival. Hepatology 2003;37(02):429-442

26 Llovet JM, Real MI, Montaña XBarcelona Liver Cancer Group, et al; Arterial embolisation or chemoembolisation versus symptomatic treatment in patients with unresectable hepatocellular carcinoma: a randomised controlled trial. Lancet 2002;359 (9319):1734-1739

27 Brown KT, Do RK, Gonen M, et al. Randomized trial of hepatic artery embolization for hepatocellular carcinoma using doxorubicin-eluting microspheres compared with embolization with microspheres alone. J Clin Oncol 2016;34(17):2046-2053

28 Malagari K, Pomoni M, Kelekis A, et al. Prospective randomized comparison of chemoembolization with doxorubicin-eluting beads and bland embolization with BeadBlock for hepatocellular carcinoma. Cardiovasc Intervent Radiol 2010;33(03):541-551

29 Lammer J, Malagari K, Vogl TPRECISION V Investigators, et al; Prospective randomized study of doxorubicin-eluting-bead embolization in the treatment of hepatocellular carcinoma: results of the PRECISION V study. Cardiovasc Intervent Radiol 2010;33(01): 41-52

30 Marelli L, Stigliano R, Triantos C, et al. Transarterial therapy for hepatocellular carcinoma: which technique is more effective? A systematic review of cohort and randomized studies. Cardiovasc Intervent Radiol 2007;30(01):6-25

31 Zhang R, Shen L, Zhao L, Guan Z, Chen Q Li W. Combined transarterial chemoembolization and microwave ablation versus transarterial chemoembolization in BCLC stage B hepatocellular carcinoma. Diagn Interv Radiol 2018;24(04):219-224

32 Xu LF, Sun HL, Chen YT, et al. Large primary hepatocellular carcinoma: transarterial chemoembolization monotherapy versus combined transarterial chemoembolization-percutaneous microwave coagulation therapy. J Gastroenterol Hepatol 2013;28 (03):456-463

33 Zheng L, Li HL, Guo CY, Luo SX. Comparison of the efficacy and prognostic factors of transarterial chemoembolization plus microwave ablation versus transarterial chemoembolization alone in patients with a large solitary or multinodular hepatocellular carcinomas. Korean J Radiol 2018;19(02):237-246

34 Yang Y, Si T. Yttrium-90 transarterial radioembolization versus conventional transarterial chemoembolization for patients with hepatocellular carcinoma: a systematic review and meta-analysis. Cancer Biol Med 2018;15(03):299-310

35 Salem R, Gordon AC, Mouli S, et al. Y90 radioembolization significantly prolongs time to progression compared with chemoembolization in patients with hepatocellular carcinoma. Gastroenterology 2016;151(06):1155-1163.e2
36 Yao FY, Mehta N, Flemming J, et al. Downstaging of hepatocellular cancer before liver transplant: long-term outcome compared to tumors within Milan criteria. Hepatology 2015;61(06): 1968-1977

37 Zhu XD, Sun HC. Emerging agents and regimens for hepatocellular carcinoma. J Hematol Oncol 2019;12(01):110

38 Finn RS, Qin S, Ikeda MIMbrave150 Investigators, et al; Atezolizumab plus bevacizumab in unresectable hepatocellular carcinoma. N Engl J Med 2020;382(20):1894-1905

39 Kudo M, Han G, Finn RS, et al. Brivanib as adjuvant therapy to transarterial chemoembolization in patients with hepatocellular carcinoma: A randomized phase III trial. Hepatology 2014;60 (05):1697-1707

40 Lencioni R, Llovet JM, Han G, et al. Sorafenib or placebo plus TACE with doxorubicin-eluting beads for intermediate stage HCC: The SPACE trial. J Hepatol 2016;64(05):1090-1098

41 Kok VC, Chen YC, Chen YY, et al. Sorafenib with transarterial chemoembolization achieves improved survival vs. sorafenib alone in advanced hepatocellular carcinoma: a nationwide population-based cohort study. Cancers (Basel) 2019;11(07):E985

42 Park JW, Kim YJ, Kim DY, et al. Sorafenib with or without concurrent transarterial chemoembolization in patients with advanced hepatocellular carcinoma: the phase III STAH trial. J Hepatol 2019;70(04):684-691

43 Zhao Y, Wang WJ, Guan S, et al. Sorafenib combined with transarterial chemoembolization for the treatment of advanced hepatocellular carcinoma: a large-scale multicenter study of 222 patients. Ann Oncol 2013;24(07):1786-1792

44 Chen S, Yu W, Zhang K, Liu W. Comparison of the efficacy and safety of Transarterial chemoembolization with and without Apatinib for the treatment of BCLC stage C hepatocellular carcinoma. BMC Cancer 2018;18(01):1131

45 Varghese J, Kedarisetty C, Venkataraman J, et al. Combination of TACE and sorafenib improves outcomes in BCLC stages B/C of hepatocellular carcinoma: a single centre experience. Ann Hepatol 2017;16(02):247-254

46 Vilgrain V, Pereira H, Assenat ESARAH Trial Group, et al; Efficacy and safety of selective internal radiotherapy with yttrium-90 resin microspheres compared with sorafenib in locally advanced and inoperable hepatocellular carcinoma (SARAH): an open-label randomised controlled phase 3 trial. Lancet Oncol 2017;18(12): 1624-1636

47 Gandhi M, Choo SP, Thng CHAsia-Pacific Hepatocellular Carcinoma Trials Group, et al. Single administration of selective internal radiation therapy versus continuous treatment with sorafeNIB in locally advanced hepatocellular carcinoma (SIRveNIB): study protocol for a phase iii randomized controlled trial. BMC Cancer 2016;16(01):856

48 Garin E, Tzelikas L, Guiu B, et al. Major impact of personalized dosimetry using 90Y loaded glass microspheres SIRT in HCC: final overall survival analysis of a multicenter randomized phase II study (DOSISPHERE-01). J Clin Oncol 2020;38(04):516

49 Chaudhry M, McGinty KA, Mervak B, et al. The LI-RADS version 2018 MRI treatment response algorithm: evaluation of ablated hepatocellular carcinoma. Radiology 2020;294(02):320-326 